\title{
Galactic Escape Speeds in Mirror and Cold Dark Matter Models
}

\author{
Alvin J. K. Chud ${ }^{1}$, D. T. Wickramasinghe ${ }^{1}$, Lilia Ferrarid ${ }^{\sqrt{a \mid}}$ \\ ${ }^{1}$ Mathematical Sciences Institute, The Australian National University, ACT 0200, Australia
}

Received: date / Accepted: date

\begin{abstract}
The mirror dark matter (MDM) model of Berezhiani et al. has been shown to reproduce observed galactic rotational curves for a variety of spiral galaxies, and has been presented as an alternative to cold dark matter (CDM) models. We investigate possible additional tests involving the properties of stellar orbits, which may be used to discriminate between the two models. We demonstrate that in MDM and CDM models fitted equally well to a galactic rotational curve, one generally expects predictable differences in escape speeds from the disc.

The recent radial velocity (RAVE) survey of the Milky Way has pinned down the escape speed from the solar neighbourhood to $v_{\mathrm{esc}}=544_{-46}^{+64} \mathrm{~km} \mathrm{~s}^{-1}$, placing an additional constraint on dark matter models. We have constructed an MDM model for the Milky Way based on its rotational curve, and find an escape speed that is just consistent with the observed value given the current errors, which lends credence to the viability of the MDM model. The Gaia-ESO spectroscopic survey is expected to lead to an even more precise estimate of the escape speed that will further constrain dark matter models. However, the largest differences in stellar escape speeds between both models are predicted for dark matter dominated dwarf galaxies such as DDO 154, and kinematical studies of such galaxies could prove key in establishing, or abolishing, the validity of the MDM model.
\end{abstract}

\section{Introduction}

Over the last decade, mirror matter has emerged as a promising candidate for dark matter. The idea that there might be a hidden "mirror sector" of the Universe

a e-mail: Lilia.Ferrario@anu.edu.au dates back to [27, was subsequently expanded upon by 22], and owes its modern form (in the context of gauge theories) to [18. It is based on the observation that parity symmetry is violated by the weak interaction in the "ordinary" sector we observe, but may be restored by the existence of a duplicate sector with the same fundamental particles and microphysics (apart from the opposite handedness of the weak interaction). Support for the existence of a mirror world may also come from the recent experiments of [6] on neutron oscillations.

Mirror dark matter (MDM) models linked to superstring inspired unifications of the four forces in the early universe have been discussed by 23 . The ordinary and mirror sectors are coupled through gravity but not the other fundamental interactions, which explains the intrinsic suitability of mirror matter as a dark matter candidate. Much research has been done on cosmological signatures of mirror matter; these turn up in the context of early Universe thermodynamics, Big Bang nucleosynthesis, primordial structure formation/evolution, cosmic microwave background (CMB) radiation and large-scale structure power spectra. Detailed reviews of this work have been provided by 3 and [14].

Astrophysical signatures of mirror matter have also been looked at in the context of galactic rotational curves, using a specific model for mirror gravity developed by [32, 4] and [5] (henceforth BPR). In this model, the ordinary and mirror sectors are assumed to have separate metric tensors (which are coupled to a third metric tensor), leading to a Yukawa-like modification of the gravitational interaction between both sectors. An ordinary matter test particle located at a radial distance $r$ from a point-like source of ordinary mass $M_{1}$ and mirror mass $M_{2}$ will feel a Yukawa-like 
potential

$\phi(r)=-\frac{G}{2 r}\left(M_{1}+M_{2}+\left(M_{1}-M_{2}\right) e^{-\frac{r}{r_{m}}}\right)$,

where $G$ is the Newtonian gravitational constant and $r_{m}$ is the Yukawa radius. The free-fall acceleration of the particle follows as

$g(r)=\frac{G}{2 r^{2}}\left[M_{1}+M_{2}+\left(M_{1}-M_{2}\right)\left(1+\frac{r}{r_{m}}\right) e^{-\frac{r}{r_{m}}}\right]$.

Although mirror matter is unable to form extended halos around galaxies due to its collisional and dissipative nature, the combined model of mirror matter and mirror gravity has been successfully fitted to the observed rotational curves of several disc galaxies by BPR, on the assumption that ordinary matter has a similar density profile to its mirror counterpart. This makes it the most viable theory of MDM that is presently available.

In this paper, we investigate some implications of the BPR model over and above its demonstrated success in modelling rotation curves, and suggest astrophysical signatures that may be observable and potentially used to discriminate between the MDM and CDM models. In Section 2, we use point-like source models to investigate the orbits of ordinary matter particles in the presence of mirror matter, and show that significant differences are expected in the nature of orbits near the Yukawa radius. We then construct in Section $3 \mathrm{CDM}$ models for the galaxies NGC 2403, DDO 170 and DDO 154 (whose rotational curves have previously been modelled using MDM), and calculate and compare escape speeds from the stellar disc as a function of galactic radius for both types of model. We show that the MDM models predict specific values for the escape speeds that are generally different from those in the CDM models. In Section 4, we construct MDM and CDM models for the Milky Way, and find that the escape speed from the solar neighbourhood in the MDM model is in good agreement with the current observed value. We discuss and present our main conclusions in Section 5.

\section{Point-like source orbits}

The deviation of mirror gravity from an inverse-square law leads to distinctive orbital dynamics in the gravitational field of a point-like source, especially at distances $r \sim r_{m}$. Two prominent features are orbital precession and a varying escape speed to circular speed ratio, neither of which are present for point-like sources in classical gravity.
Orbits for an ordinary test body in the gravitational field given by Equations (11) and (2) are described by its Binet equation

$\frac{d^{2} u}{d \theta^{2}}+u=$

$\frac{G}{2 h^{2}}\left[M_{1}+M_{2}+\left(M_{1}-M_{2}\right)\left(1+\frac{1}{r_{m} u}\right) e^{-\frac{1}{r_{m} u}}\right]$,

where the reciprocal radial distance $u=1 / r$ is a function of the plane polar angle $\theta$, and the specific angular momentum $h=L / m=r^{2} \dot{\theta}$ is a conserved quantity. Taking the Yukawa radius $r_{m}=10 \mathrm{kpc}$ (as assumed by BPR) here and henceforth, we solve Equation (3) numerically for orbits about a point-like source with $M_{2} / M_{1}=10$ (see Figure 11).

The test body is given an initial radial distance of $r_{0}=\alpha r_{m}$ and an initial transverse velocity of $\mathbf{v}_{0}=$ $\sqrt{G M_{1} / r_{0}} \hat{\boldsymbol{\theta}}$, which is the circular velocity in the absence of mirror matter and mirror gravity. Its orbit is essentially Newtonian for $\alpha \ll 1$, as gravitational interaction between ordinary and mirror matter is negligible at small distances, and Equation (2) reduces to

$g(r)=\frac{G M_{1}}{r^{2}}$

in the limit $r \rightarrow 0$.

For $\alpha \gg 1$, gravitational interaction between ordinary and mirror matter becomes universal, and Equation (2) reduces to

$g(r)=\frac{G\left(M_{1}+M_{2}\right)}{2 r^{2}}$

in the limit $r \rightarrow \infty$. The test body feels the combined inverse-square field of both matter types (but with an effective gravitational constant of $G / 2$ ), resulting in an elliptical orbit.

Precessing orbits are manifest for $\alpha \sim 0.1$ to $\alpha \sim 10$, and are explicitly non-classical. They may be expected to play a role in galactic stellar dynamics at distance scales on the order of the Yukawa radius from the centre of a galaxy.

A more useful signature of mirror gravity, which may allow it to be observationally corroborated or falsified, lies in the escape speeds that it predicts. For a point-like source with gravitational field given by Equations (1) and (2), the escape and circular speeds are given respectively by

$$
\begin{aligned}
v_{\mathrm{esc}}(r) & =\sqrt{-2 \phi(r)} \\
& =\sqrt{\frac{G}{r}\left(M_{1}+M_{2}+\left(M_{1}-M_{2}\right) e^{-\frac{r}{r_{m}}}\right)},
\end{aligned}
$$



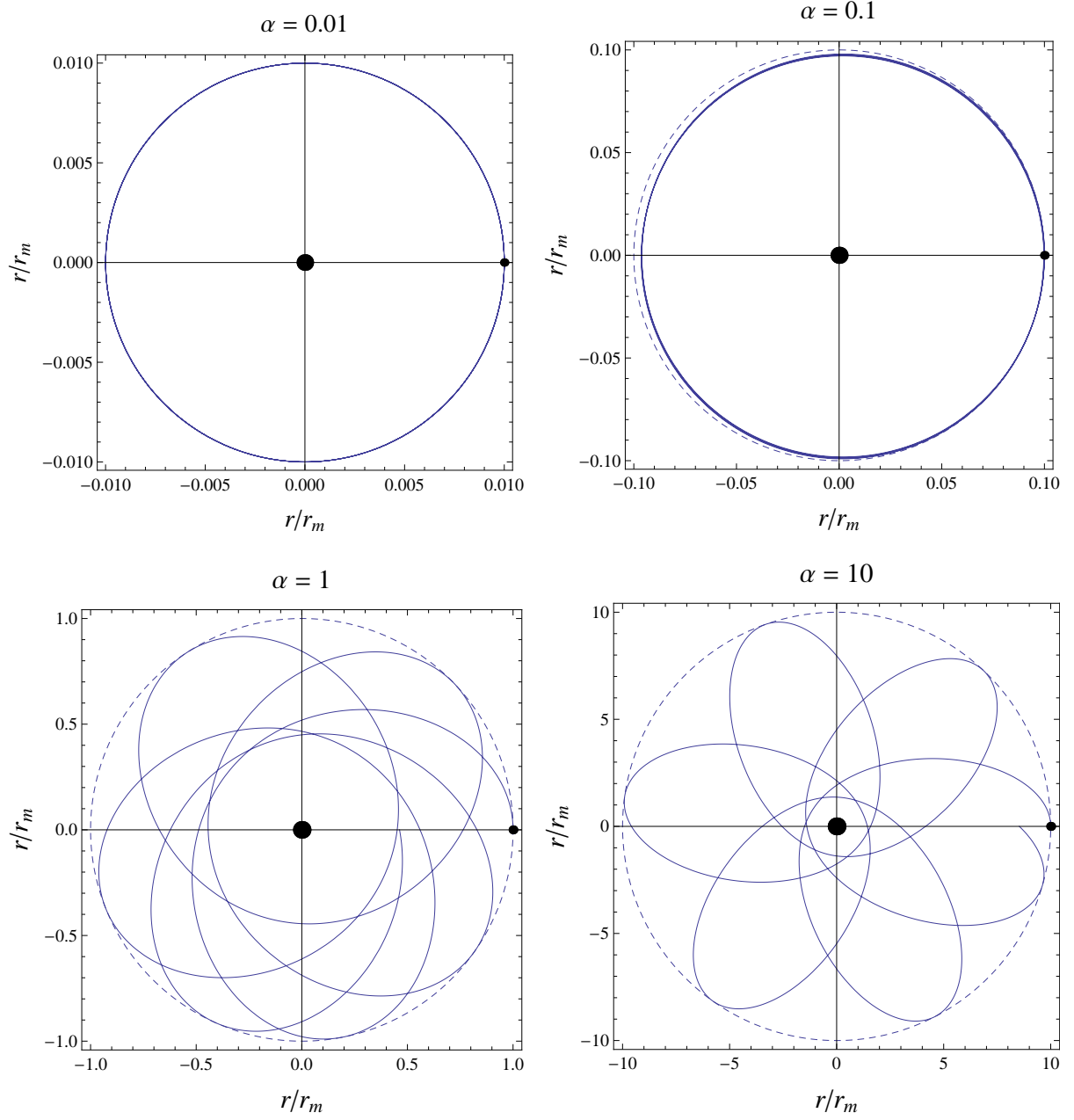

$\alpha=100$

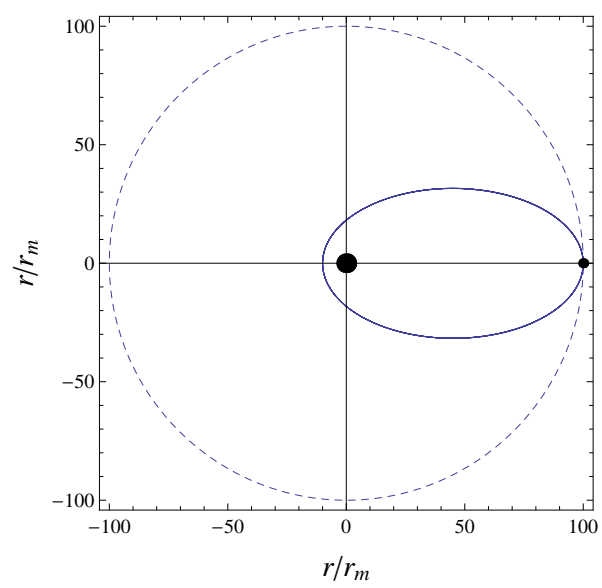

Fig. 1 Mirror gravity orbits (solid) for ordinary test body about point-like source with $M_{2} / M_{1}=10$, initial radius $r_{0}=\alpha r_{m}$ and initial velocity $\mathbf{v}_{0}=\sqrt{G M_{1} / r_{0}} \hat{\boldsymbol{\theta}}$. The corresponding Newtonian orbits (dashed) are circular. All orbits are shown up to five revolutions. 


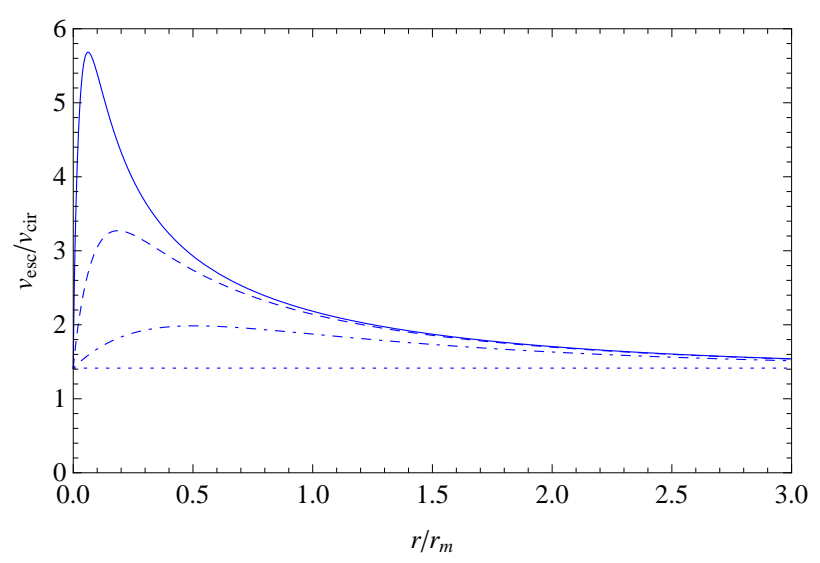

Fig. 2 Plot of $v_{\text {esc }} / v_{\text {cir }}$ against $r / r_{m}$ for $M_{2} / M_{1}=1000$ (solid), $M_{2} / M_{1}=100$ (dashed) and $M_{2} / M_{1}=10$ (dotdashed). The Newtonian ratio $v_{\text {esc }} / v_{\text {cir }}=\sqrt{2}$ is recovered for $M_{2} / M_{1}=1$ (dotted).

and

$$
\begin{aligned}
& v_{\text {cir }}(r)=\sqrt{r g(r)} \\
& \quad=\sqrt{\frac{G}{2 r}\left[M_{1}+M_{2}+\left(M_{1}-M_{2}\right)\left(1+\frac{r}{r_{m}}\right) e^{\left.-\frac{r}{r_{m}}\right]}\right.} .
\end{aligned}
$$

In general, the ratio $v_{\text {esc }} / v_{\text {cir }} \neq \sqrt{2}$ and is a function of $r$, in contrast to the Newtonian case. A plot of $v_{\text {esc }} / v_{\text {cir }}$ for different values of $M_{2} / M_{1} \geq 1$ shows that $v_{\text {esc }} / v_{\text {cir }} \geq \sqrt{2}$, with the discrepancy being more pronounced for larger mass ratios (see Figure 2). 11 This suggests that mirror gravity may predict significantly larger escape speeds compared to classical gravity when both models are fitted to give similar circular speeds, as is done in the modelling of galactic rotational curves.

\section{Escape speeds in disc galaxies}

Mirror matter and mirror gravity have been put forth by $\mathrm{BPR}$ as a collisional and dissipative dark matter candidate capable of explaining flat galactic rotational curves. They have successfully fitted this MDM model to the observed rotational curves of several disc galaxies, using simplified and similar mass density profiles for the visible and dark matter distributions. In this section, we obtain comparable fits using a CDM model of the same complexity, and generate the corresponding galactic escape speed curves for both models.

\footnotetext{
${ }^{1}$ The cosmological ratio between the dark and visible energy densities is $\rho_{2} / \rho_{1} \approx 10$ in the BPR model. $M_{2} / M_{1}$ should approach the cosmological ratio for larger galaxies, while smaller galaxies are expected to be more dark matter-dominated. In any case, only values of $M_{2} / M_{1}>1$ will be considered in this paper.
}

The distribution of visible matter in a typical disc galaxy is approximated most simply as a two-dimensional disc with the exponential surface mass density

$\Sigma_{1, d}(r)=\frac{M_{1, d}}{2 \pi r_{1, d}^{2}} e^{-\frac{r}{r_{1, d}}}$,

where $M_{1, d}$ and $r_{1, d}$ are the total mass and characteristic length scale of the disc respectively. In the BPR model, the distribution of mirror dark matter is assumed to take on a similar profile (i.e. another exponential disc concentric to the visible disc); this is justifiable since mirror matter has the same microphysics as ordinary matter and can be expected to undergo the same process of galaxy formation, up to a rescaling of parameters. Hence its surface mass density is given by

$\Sigma_{2, d}(r)=\frac{M_{2, d}}{2 \pi r_{2, d}^{2}} e^{-\frac{r}{r_{2, d}}}$,

where $M_{2, d}$ and $r_{2, d}$ are the corresponding parameters for the mirror dark disc.

For an ordinary test body with position vector $\mathbf{r}$ in the plane of the two discs, the acceleration vector follows as

$$
\begin{aligned}
& \mathbf{g}(\mathbf{r})=-\frac{G}{2} \int_{\operatorname{disc}} d^{2} \mathbf{r}^{\prime} \frac{\mathbf{r}-\mathbf{r}^{\prime}}{\left|\mathbf{r}-\mathbf{r}^{\prime}\right|^{3}}\left[\Sigma_{1, d}\left(r^{\prime}\right)+\Sigma_{2, d}\left(r^{\prime}\right)\right. \\
& \left.+\left(\Sigma_{1, d}\left(r^{\prime}\right)-\Sigma_{2, d}\left(r^{\prime}\right)\right)\left(1+\frac{\left|\mathbf{r}-\mathbf{r}^{\prime}\right|}{r_{m}}\right) e^{-\frac{\left|\mathbf{r}-\mathbf{r}^{\prime}\right|}{r_{m}}}\right]
\end{aligned}
$$

where $\mathbf{g}(\mathbf{r})=-g(r) \hat{\mathbf{r}}$. The integration can be performed semi-analytically in this case, and yields the rotational curve $v(r)=\sqrt{r g(r)}$ of the model.

Assuming that the mass-to-light ratio $\gamma=M_{1, d} / M_{L}$ of the visible matter (where the luminous mass $M_{L}$ is an observed fixed parameter) is constant for each galaxy, the dimensionless quantities $\gamma, \beta=M_{2, d} / M_{1, d}$ and $\alpha=r_{2, d} / r_{1, d}$ have been taken by BPR as fit parameters for the rotational curves of four disc galaxies. 2 The visible length scale $r_{1, d}$ for each galaxy is deduced from and fixed by observations. The resulting threeparameter fits are demonstrably good, with $\chi_{\text {red }}^{2} \approx 1$ for each galaxy (see Figure 3 and Table 1).

Numerous galactic rotational curve fits for CDM models exist and are well documented in the literature. These are of varying complexity, but nearly all of them incorporate components such as a galactic bulge or a gaseous contribution, in addition to the standard

${ }^{2}$ Rotational curve fits for the large spiral galaxy NGC 7331 require the contribution of an additional bulge component, and will be omitted from the MDM-CDM(IT) comparisons in this section. We address the issue of a galactic bulge when modelling the Milky Way in Section 4. 
galactic disc and dark halo components. Indeed, the rotational curves of the galaxies considered by BPR have been fitted for a multi-component CDM model by [2] (henceforth BBS).

We adopt a simplified two-component version of the BBS model in this paper, allowing direct comparison between the MDM model and a CDM model of the same complexity. The distribution of visible matter is again an exponential disc with surface mass density given by Equation (6), while a pseudo-isothermal truncated sphere (which we shall refer to from now on as $\mathrm{CDM}(\mathrm{IT})$ ) is assumed for the distribution of dark matter.

The volume mass density of the dark halo is given by

$\rho_{h}(r)=\frac{\rho_{s}}{1+\frac{r^{2}}{r_{s}^{2}}}$,

where $\rho_{s}$ and $r_{s}$ are the central density and characteristic length scale of the halo respectively. This profile avoids the central cusps inherent in those produced by numerical $n$-body simulations (e.g. the model by [31]), but has a divergent total mass and must be truncated at a suitable distance from the galactic centre.

An analytical expression is available for the rotational curve of this simplified BBS model. We can write

$v(r)=\sqrt{v_{\text {disc }}^{2}(r)+v_{\text {halo }}^{2}(r)}$,

where $v_{\text {disc }}(r)$ and $v_{\text {halo }}(r)$ are rotational curves for the visible disc and dark halo respectively. The contribution from the disc has been obtained by [19], using a method by 35 , as

$$
\begin{aligned}
v_{\text {disc }}^{2}(r) & =\frac{G M_{1, d} r^{2}}{2 r_{1, d}^{3}}\left[I_{0}\left(\frac{r}{2 r_{1, d}}\right) K_{0}\left(\frac{r}{2 r_{1, d}}\right)\right. \\
& \left.-I_{1}\left(\frac{r}{2 r_{1, d}}\right) K_{1}\left(\frac{r}{2 r_{1, d}}\right)\right],
\end{aligned}
$$

where the $I_{n}$ and $K_{n}$ are modified Bessel functions evaluated at $r / 2 r_{1, d}$. 1 gives the contribution from the halo as

$v_{\text {halo }}^{2}(r)=v_{\max }^{2}\left(1-\frac{r_{s}}{r} \tan ^{-1} \frac{r}{r_{s}}\right)$,

where $v_{\max }=\sqrt{4 \pi G \rho_{s} r_{s}^{2}}$ is the asymptotic rotational speed for the halo at $r \gg r_{s}$.

As in the BPR model, we use data from [1, 12, [25] and [2] for the intermediate spiral galaxy NGC 2403 and the dwarf galaxies DDO 170 and DDO 154. Again assuming a constant mass-to-light ratio for each galaxy and taking $M_{L}$ and $r_{1, d}$ as fixed parameters, we fit the rotational curves of these three disc galaxies by using $\gamma, v_{\max }$ and $r_{s}$ as fit parameters.
Furthermore, we have generated synthetic rotational curves for two disc galaxies, one whose physics is dictated by MDM and the other by CDM(IT). Typical observational errors (Gaussian noise) have been assigned to these synthetic galaxies, which are then treated as actual observed galaxies and fitted using the procedures outlined in this section. We find that the MDM fit recovers best-fit values in good agreement with the actual values for the synthetic MDM galaxy, while the CDM(IT) fit matches the parameters of the synthetic CDM(IT) galaxy as well; this verifies the accuracy of our fitting algorithms. Finally, a side-by-side comparison of all the MDM and CDM(IT) fits shows that both models are able to reproduce both the observed and synthetic galactic rotational curves, and equally well (see Figure 3 and Table 1).

Now, for the MDM model, the gravitational potential felt by an ordinary test body with position vector $\mathbf{r}$ in the plane of the two discs is given by

$$
\begin{aligned}
\phi_{\mathrm{MDM}}(r) & =-\frac{G}{2} \int_{\mathrm{disc}} d^{2} \mathbf{r}^{\prime} \frac{1}{\left|\mathbf{r}-\mathbf{r}^{\prime}\right|}\left[\Sigma_{1, d}\left(r^{\prime}\right)+\Sigma_{2, d}\left(r^{\prime}\right)\right. \\
& \left.+\left(\Sigma_{1, d}\left(r^{\prime}\right)-\Sigma_{2, d}\left(r^{\prime}\right)\right) e^{-\frac{\left|\mathbf{r}-\mathbf{r}^{\prime}\right|}{r_{m}}}\right]
\end{aligned}
$$

where $\phi_{\mathrm{MDM}}(r)$ depends only on $r$ due to cylindrical symmetry. For the CDM(IT) model, the gravitational potential felt by a test body with position vector $\mathbf{r}$ in the plane of the disc is given by

$\phi_{\mathrm{CDM}(\mathrm{IT})}(r)=-G \int_{\text {disc }} d^{2} \mathbf{r}^{\prime} \frac{\Sigma_{1, d}\left(r^{\prime}\right)}{\left|\mathbf{r}-\mathbf{r}^{\prime}\right|}-G \int_{\text {halo }} d^{3} \mathbf{r}^{\prime} \frac{\rho_{h}\left(r^{\prime}\right)}{\left|\mathbf{r}-\mathbf{r}^{\prime}\right|}$,

where $\phi_{\mathrm{CDM}(\mathrm{IT})}(r)$ depends only on $r$ as well.

Using the parameters given in Table 1] we obtain MDM and CDM(IT) escape speed curves $v(r)=\sqrt{-2 \phi(r)}$ for the five disc galaxies by numerical integration, truncating the discs and halos at a value of $r$ just beyond the farthest data point for each galaxy (see Figure 4).

As anticipated from our discussion of point-like source models in Section 2, escape speeds for stars in the galactic plane are mostly higher in the MDM model. The offset between the two models appears to be nearly constant out to the truncation distance. It is both absolutely and relatively larger for the dwarf galaxies $(\Delta v \geq$ $30 \mathrm{kms}^{-1}$ and $\left.\Delta v / v_{\mathrm{CDM}(\mathrm{IT})} \geq 0.2\right)$ than for NGC 2403 $\left(\Delta v \approx 10 \mathrm{kms}^{-1}\right.$ and $\left.\Delta v / v_{\mathrm{CDM}(\mathrm{IT})} \approx 0.03\right)$.

This systematic escape speed discrepancy may be attributed to the increasing importance of mirror dark matter over ordinary matter in the smaller galaxies (as 

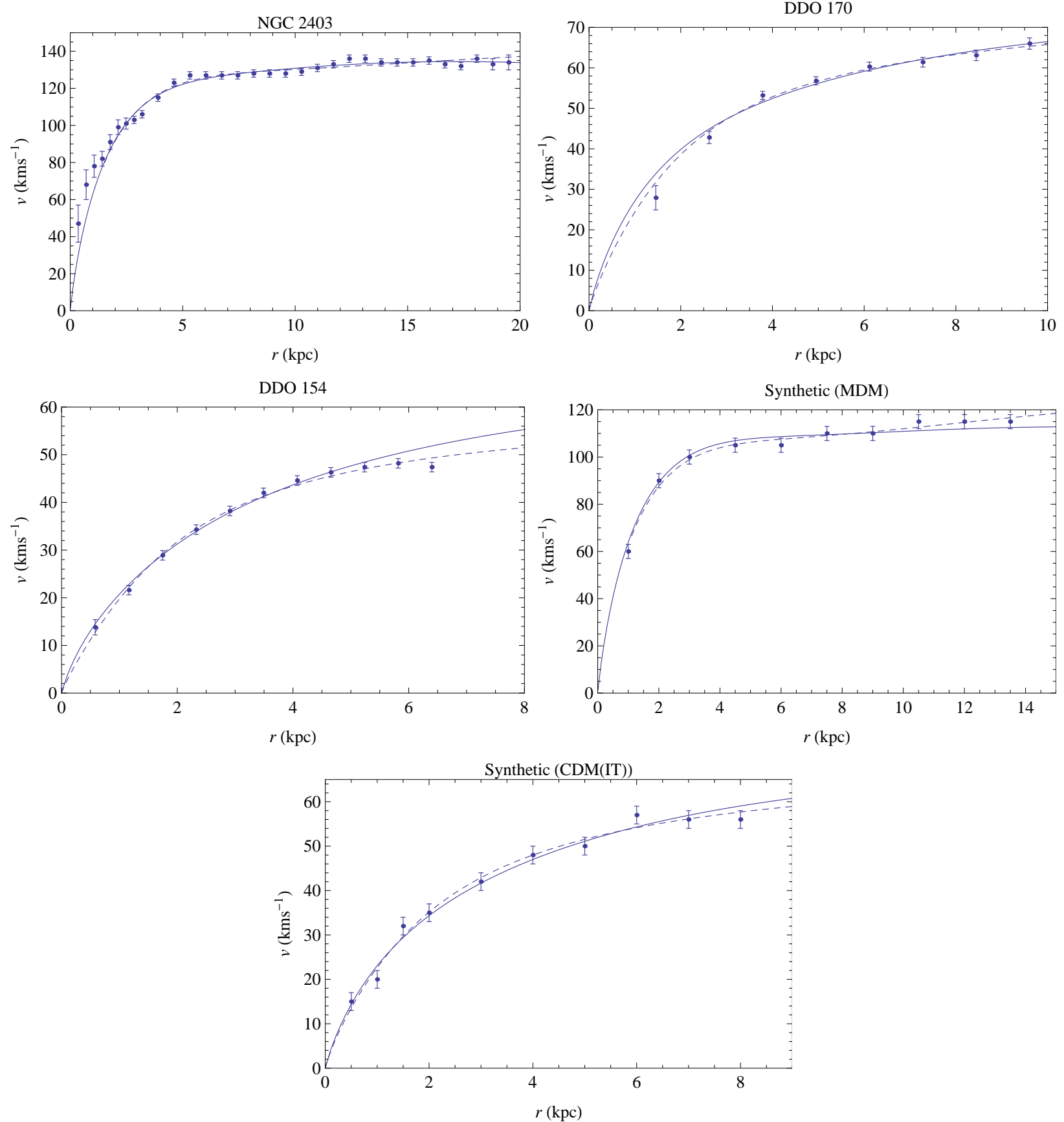

Fig. 3 Observed and synthetic rotational curves for different disc galaxies, with both MDM (solid) and CDM(IT) (dashed) three-parameter fits. Parameters and values of $\chi_{\text {red }}^{2}$ are given in Table 1

indicated by the higher values of $\beta$ ), which in turn is due to typical distance scales in these galaxies being smaller than the Yukawa scale $r_{m}(\sim 10 \mathrm{kpc})$ such that dark matter is less effective in gravitationally binding to ordinary matter. In the largest galaxies such as the Milky Way (see Section 4), the ratio of the energy density of mirror dark matter to visible matter $\rho_{2} / \rho_{1}$ ap- proaches the cosmological value $\sim 10$ as required by the BPR model.

Crucially, we have also observed in our analysis that escape speeds for the MDM model do not depend significantly on the choice of truncation radius, and are constrained by the rotational curve fits. Thus, observations of the stellar escape speeds in disc galaxies of different 
Table $1 \mathrm{MDM}$ and $\mathrm{CDM}(\mathrm{IT})$ best-fit parameters for the rotational curves of different disc galaxies. $M_{L}$ and $r_{1, d}$ are taken as fixed parameters, while $\gamma, \beta, \alpha, v_{\max }$ and $r_{s}$ are taken as fit parameters. Both models provide realistic (all values of $\gamma \sim 1$ ) and good (all values of $\chi_{\text {red }}^{2} \approx 1$ ) fits. The fitting algorithms recover best-fit values in good agreement with the actual values for the synthetic disc galaxies (given in parentheses).

\begin{tabular}{|c|c|c|c|c|c|c|}
\hline & & NGC 2403 & DDO 170 & DDO 154 & Synthetic (MDM) & Synthetic (CDM(IT)) \\
\hline \multirow[t]{2}{*}{ Fixed parameters } & $M_{L}\left(10^{9} M_{\odot}\right)$ & 7.9 & 0.18 & 0.05 & 8.0 & 0.10 \\
\hline & $r_{1, d}(\mathrm{kpc})$ & 2.0 & 1.1 & 0.5 & 1.6 & 0.8 \\
\hline \multirow[t]{7}{*}{ MDM fit } & $\gamma=M_{1, d} / M_{L}$ & 1.6 & 2.2 & 0.6 & $1.0(1.0)$ & 1.2 \\
\hline & $M_{1, d}\left(10^{9} M_{\odot}\right)$ & 12.6 & 0.4 & 0.03 & $8.2(8.0)$ & 0.12 \\
\hline & $\beta=M_{2, d} / M_{1, d}$ & 20.6 & 190 & 2000 & $22.3(23.0)$ & 567 \\
\hline & $M_{2, d}\left(10^{9} M_{\odot}\right)$ & 260 & 76 & 60 & $183(184)$ & 68 \\
\hline & $\alpha=r_{2, d} / r_{1, d}$ & 1.6 & 0.8 & 1.3 & $1.6(1.6)$ & 1.0 \\
\hline & $r_{2, d}(\mathrm{kpc})$ & 3.2 & 0.87 & 0.66 & $2.5(2.6)$ & 0.8 \\
\hline & $\chi_{\mathrm{red}}^{2}$ & 1.3 & 1.5 & 0.6 & 1.3 & 1.3 \\
\hline \multirow[t]{5}{*}{$\mathrm{CDM}(\mathrm{IT}) \mathrm{fit}$} & $\gamma=M_{1, d} / M_{L}$ & 1.5 & 1.1 & 0.2 & 1.0 & $0.9(1.0)$ \\
\hline & $M_{1, d}\left(10^{9} M_{\odot}\right)$ & 12.1 & 0.2 & 0.01 & 7.7 & $0.09(0.10)$ \\
\hline & $v_{\max }\left(\mathrm{kms}^{-1}\right)$ & 155 & 77 & 61 & 129 & $70(70)$ \\
\hline & $r_{s}(\mathrm{kpc})$ & 5.0 & 2.1 & 1.7 & 4.1 & $2.0(2.0)$ \\
\hline & $\chi_{\text {red }}^{2}$ & 1.6 & 1.2 & 0.8 & 1.3 & 1.0 \\
\hline
\end{tabular}

sizes could prove key in establishing - or abolishingthe validity of the MDM model.

\section{Escape speeds in the Milky Way}

Although the rotational curves of numerous external disc galaxies have been obtained to a good degree of precision, there are no direct estimates of their escape speeds at the present time. Conversely, while the rotational curve of our own galaxy is less established, efforts have been made by 34] to constrain the escape speed at the solar neighbourhood through the Radial Velocity Experiment (RAVE) survey. The current estimate is significantly in excess of $\sqrt{2} v_{\text {cir }}$ (where $v_{\text {cir }} \approx$ $220 \mathrm{kms}^{-1}$ is the local circular speed), and has been taken as independent evidence in favour of CDM models. In this section, we repeat the MDM-CDM(IT) comparison for the Milky Way, and show that the MDM model is also able to predict a local escape speed comparable to the currently observed value.

As in Section 3. the visible disc of the Galaxy in both models is taken as an exponential disc and described by Equation (6). Likewise, the density profiles of the mirror dark disc in the MDM model and the dark halo in the CDM(IT) model are given respectively by Equations (7) and (9). In both models, however, an additional bulge component is required to reproduce the observed Galactic rotational curve.
We employ the tractable Plummer sphere for this purpose, in lieu of the truncated power-law bulges used in more detailed mass models. Hence the gravitational potential of the visible bulge is given by

$$
\phi_{\text {bulge }}(r)=-\frac{G M_{1, b}}{\sqrt{r^{2}+r_{1, b}^{2}}},
$$

where $M_{1, b}$ and $r_{1, b}$ are the total mass and characteristic length scale of the bulge respectively. Its volume mass density follows as

$\rho_{1, b}(r)=\frac{3 M_{1, b}}{4 \pi r_{1, b}^{3}}\left(1+\frac{r^{2}}{r_{1, b}^{2}}\right)^{-\frac{5}{2}}$

from Poisson's equation.

By assumption, the presence of a visible bulge necessitates the addition of a mirror dark bulge component to the MDM model. This is similarly described by

$\rho_{2, b}(r)=\frac{3 M_{2, b}}{4 \pi r_{2, b}^{3}}\left(1+\frac{r^{2}}{r_{2, b}^{2}}\right)^{-\frac{5}{2}}$

where $M_{2, b}$ and $r_{2, b}$ are the corresponding parameters for the dark bulge. The acceleration vector of an ordinary test body with position vector $\mathbf{r}$ in the Galactic plane follows as

$\mathbf{g}(\mathbf{r})=\mathbf{g}_{\text {disc }}(\mathbf{r})+\mathbf{g}_{\text {bulge }}(\mathbf{r})$, 

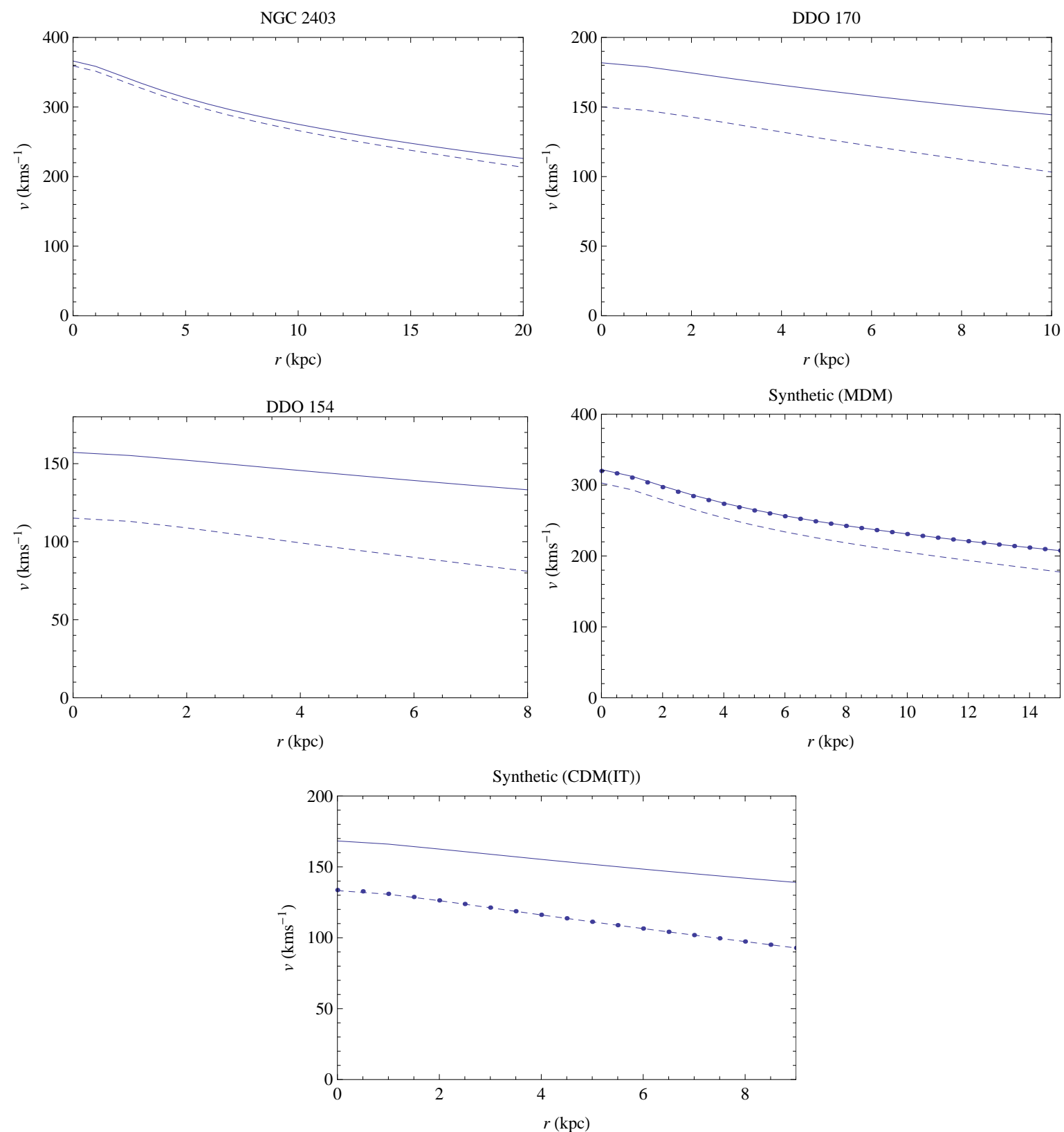

Fig. 4 Predicted MDM (solid) and CDM(IT) (dashed) escape speed curves for stars in the galactic plane of observed and synthetic disc galaxies, using parameters given in 1 The actual (dotted) escape speed curves for the synthetic galaxies have been included as well.

where $\mathbf{g}_{\text {disc }}(\mathbf{r})$ is given by Equation (8) and $\mathbf{g}_{\text {bulge }}(\mathbf{r})$ is obtained in identical fashion by numerically integrating Equations (16) and (17) over the bulge.

With the addition of the visible bulge, the rotational curve of the CDM(IT) model becomes

$v(r)=\sqrt{v_{\text {disc }}^{2}(r)+v_{\text {halo }}^{2}(r)+v_{\text {bulge }}^{2}(r)}$,

where $v_{\text {disc }}^{2}(r)$ and $v_{\text {halo }}^{2}(r)$ are given by Equations (11) and (12) as before, and $v_{\text {bulge }}^{2}(r)$ follows from Equation
(15) as

$v_{\text {bulge }}^{2}(r)=r \frac{d}{d r} \phi_{\text {bulge }}(r)=\frac{G M_{1, b} r^{2}}{r_{1, b}^{3}}\left(1+\frac{r^{2}}{r_{1, b}^{2}}\right)^{-\frac{3}{2}}$.

For the MDM model, we assume a constant darkto-visible mass ratio (such that $\beta=M_{2, i} / M_{1, i}$ ) and a constant dark-to-visible length scale ratio (such that $\left.\alpha=r_{2, i} / r_{1, i}\right)$ in the Galaxy. We use $M_{1, d}, M_{1, b}, \beta$ and 


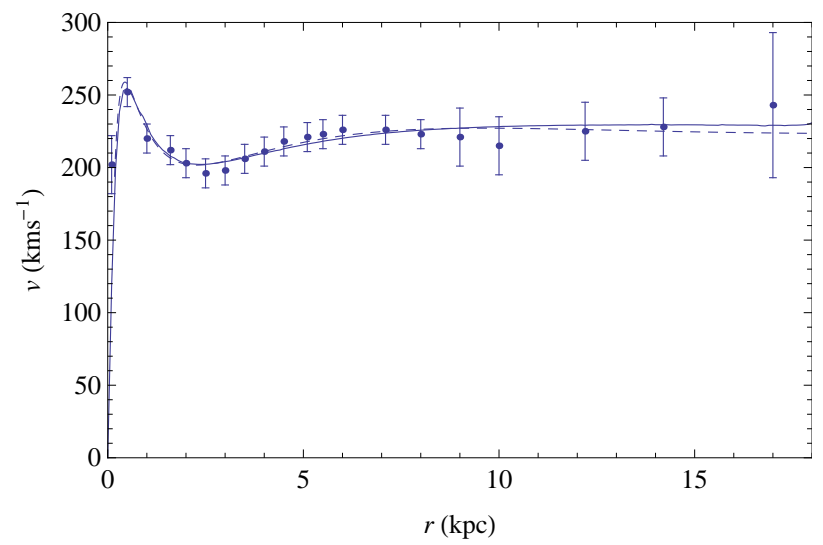

Fig. 5 Observed Galactic rotational curve out to $2 R_{\odot}$, with both MDM (solid) and CDM(IT) (dashed) four-parameter fits. Parameters and values of $\chi_{\text {red }}^{2}$ are given in Table 2 .

Table $2 \mathrm{MDM}$ and CDM(IT) best-fit parameters for the Galactic rotational curve out to $2 R_{\odot} \cdot r_{1, d}$ and $r_{1, b}$ are taken as fixed parameters, while $M_{1, d}, M_{1, b}, \beta, \alpha, v_{\max }$ and $r_{s}$ are taken as fit parameters. Both models provide good $\left(\chi_{\text {red }}^{2} \approx 1\right)$ fits that agree with observation and other mass models.

\begin{tabular}{ccc}
\hline & Parameters & Values \\
\hline Fixed parameters & $r_{1, d}(\mathrm{kpc})$ & 3 \\
& $r_{1, b}(\mathrm{kpc})$ & 0.3 \\
MDM fit & $M_{1, d}\left(10^{9} M_{\odot}\right)$ & 48 \\
& $M_{1, b}\left(10^{9} M_{\odot}\right)$ & 13 \\
& $\beta=M_{2, i} / M_{1, i}$ & 12 \\
& $M_{2, d}\left(10^{9} M_{\odot}\right)$ & 576 \\
& $M_{2, b}\left(10^{9} M_{\odot}\right)$ & 156 \\
& $\alpha=r_{2, i} / r_{1, i}$ & 1.7 \\
& $r_{2, d}(\mathrm{kpc})$ & 5.1 \\
& $r_{2, b}(\mathrm{kpc})$ & 0.51 \\
$\chi_{\mathrm{red}}^{2}$ & 1.3 \\
CDM(IT) fit & $M_{1, d}\left(10^{9} M_{\odot}\right)$ & 54 \\
& $M_{1, b}\left(10^{9} M_{\odot}\right)$ & 11.8 \\
& $v_{\max }\left(\mathrm{kms}{ }^{-1}\right)$ & 241 \\
& $r_{s}(\mathrm{kpc})$ & 6.6 \\
$\chi_{\mathrm{red}}^{2}$ & 1.1 \\
\hline
\end{tabular}

$\alpha$ as fit parameters, and take $r_{1, d}=3 \mathrm{kpc}$ and $r_{1, b}=$ $0.3 \mathrm{kpc}$ in rough accordance with observation and other mass models (e.g. 29]).

For the CDM(IT) model, we use $M_{1, d}, M_{1, b}, v_{\max }$ and $r_{s}$ as fit parameters, and take $r_{1, d}$ and $r_{1, b}$ as fixed parameters with the MDM values. With data out to $2 R_{\odot}$ (where $R_{\odot}=8.5 \mathrm{kpc}$ ) from [11], 8] and [15], we are able to generate good rotational curve fits for both models (see Figure 5 and Table 2).

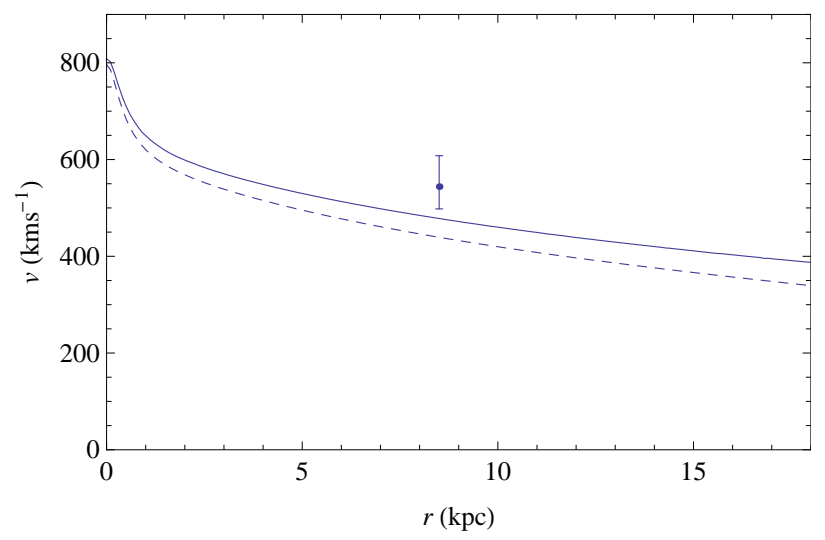

Fig. 6 Predicted MDM (solid) and CDM(IT) (dashed) escape speed curves out to $2 R_{\odot}$ for stars in the Galactic plane, using the parameters given in Tab. 2. The RAVE value $v_{\text {esc }}=544_{-46}^{+64} \mathrm{kms}^{-1}$ for the local escape speed (at $R_{\odot}=8.5 \mathrm{kpc}$ ) is included as a reference point.

Although the fits are simplistic, they are realistic enough to serve as adequate models of the Galaxy. The total visible (stellar) mass for the MDM fit is $6.1 \times$ $10^{10} M_{\odot}$ with a bulge contribution of $1.3 \times 10^{10} M_{\odot}$; these values are comparable to estimates by [20, [17], [36] and [29]. The dark-to-visible mass ratio is $\beta=12$, which approaches the cosmological ratio $\rho_{2} / \rho_{1} \approx 10$ in support of the hypothesis by BPR. The local surface mass density (at $R_{\odot}=8.5 \mathrm{kpc}$ ) of the disc is $50 M_{\odot} \mathrm{pc}^{-2}$, in good agreement with estimates by [24], [16], 7] and [17].

The total visible mass for the CDM(IT) fit is $6.6 \times$ $10^{10} M_{\odot}$ with a bulge contribution of $1.2 \times 10^{10} M_{\odot}$, while the local surface mass density of the disc is $56 M_{\odot}$ $\mathrm{pc}^{-2}$. Again, these values match up reasonably well with other estimates. The local dark matter density is $0.35 \mathrm{GeVcm}^{-3}$, which is towards the low end of bounds given by 13 and 29].

Using the parameters given in Table 2] we obtain MDM and CDM(IT) escape speed curves for the Galaxy as in Section 3. but with a bulge term added to the gravitational potential (see Figure 6). For the MDM model, $\phi_{\text {bulge }}(r)$ is obtained in identical fashion to Equation (13) by numerically integrating Equations (16) and (17) over the bulge; for the CDM(IT) model, it is given by Equation (15).

Again, escape speeds for stars in the Galactic plane are higher in the MDM model, with an offset that grows from $\approx 20 \mathrm{kms}^{-1}$ to $\approx 50 \mathrm{kms}^{-1}$ but remains nearly constant beyond $r=R_{\odot}$. The relative offset at $r=R_{\odot}$ is $\Delta v / v_{\mathrm{CDM}(\mathrm{IT})} \approx 0.09$. Both models predict values for the local escape speed that fall just short of the lower bound on the RAVE value $v_{\text {esc }}=544_{-46}^{+64} \mathrm{kms}^{-1}$; in the $\mathrm{CDM}(\mathrm{IT})$ case, the fairly large discrepancy is due to 
the truncation of the dark halo at $r=2 R_{\odot}$. Indeed, by extending the truncation radius of the halo to $45 \mathrm{kpc}$, we find an escape speed for the CDM(IT) model that matches the observed RAVE value. Regardless, the fact that the MDM model is able to predict a local value of $v_{\text {esc }}$ significantly greater than $\sqrt{2} v_{\text {cir }}$ corroborates the RAVE measurement, and lends credence to its own viability as a dark matter model.

\section{Discussion and conclusions}

The addition of a non-luminous component of matter distributed spherically about the centre of a galaxy and extending well beyond the luminous disc component (i.e. a dark matter halo) explained the flat rotational curves of disc galaxies in the framework of Newtonian gravity, and led to the CDM paradigm in cosmology. In these models, a significant fraction of the gravitating matter in the Universe is in a dark collisionless form, with a density parameter ratio between dark and baryonic matter of $\Omega_{d} / \Omega_{b} \sim 5$. The CDM model has been extremely successful in explaining early Universe thermodynamics, through to fluctuations in the CMB and the formation of structures on cosmological scales. Any viable alternative model must not only explain galactic rotational curves, but also match the successes of CDM in large-scale cosmology.

A much-discussed alternative for explaining galaxy rotational curves is the modified Newtonian dynamics (MOND) model of [28, which entertains the possibility of an acceleration due to gravity that departs from the Newtonian form in regimes where the dynamical accelerations are small. An in-depth review of the theory is provided by 33. First proposed as a phenomenological model, the essence of MOND has subsequently been incorporated in a theory of modified gravity (MOG) by [30], which is based on a covariant generalisation of Einstein's theory with the addition of auxiliary gravitational fields to the metric. MOG has been successfully used to model a large sample of galactic rotational curves [9] and galaxy clusters [10. However, these models that focus on the gravitational force appear not to be as well-placed to address questions on early Universe thermodynamics and large-scale cosmology.

On the other hand, the MDM model of BPR is based on a fundamental theory of all particles and forces; as with the CDM model, it appears to have the potential to not only explain galaxy rotational curves, but also to address problems relating to the early history of the Universe. In proposing their model, BPR point out that to date there has been no observational evidence for the dark matter postulated in CDM models. They note that one of the major unanswered questions in CDM cosmology, namely the reason for the closeness of the observed ratio $\Omega_{d} / \Omega_{b}$ to unity, may find an easier solution in MDM models - where the dark (mirror) and baryonic (ordinary) sectors are expected to be closely linked. They also note that the density profiles predicted by $n$-body simulations for the CDM halos in galaxies show a central cusp, for which there is no observational evidence from rotational curves of low surface brightness galaxies. While acknowledging that this may be a problem with the numerical simulations of galaxy formation in the CDM scenario, they point out that dark matter is collisional in the MDM model, and such cusps are not expected.

Rotational curves, being based on the circular speed (usually of gas and sometimes augmented by that of stars) in galaxies, cannot by themselves provide strong constraints on dark matter models or gravity models. Indeed, CDM, MDM and MOG models have all been successfully used to model rotational curves of both low and high surface brightness galaxies. In this paper, we have sought to highlight some of the differences that may be expected in the dynamics of stars in galactic discs, which may potentially be used to discriminate between the CDM and MDM models. In particular, we have focused on stellar escape speeds from the galactic disc. Following BPR, our basic premise is that due to the collisional and dissipative nature of MDM, matter in the two sectors will have similar density distributions up to a scaling factor. A consequence is that the MDM model has a very definite prediction on escape speeds that is readily falsifiable. Notwithstanding the uncertainties inherent in modelling the structure and extent of the CDM halos, we predict systematic and measurable differences in escape speed between the two models.

We have taken the galaxies NGC 2403, DDO 170 and DDO 154 (whose rotational curves have been modelled by BPR using MDM) along with a couple of synthetic galaxies as representative of spiral galaxies with a range of sizes, and used them to calculate and compare stellar escape speeds between the MDM and CDM(IT) models. We find that the escape speeds from the galactic disc are typically higher in the MDM model, with the percentage increase in escape speed relative to what is expected from an equivalent CDM(IT) model approaching $\Delta v / v_{\mathrm{CDM}} \geq 20$ percent in the smaller low surface brightness galaxies. A similar procedure has been adopted for the Milky Way, where in addition to the MDM stellar disc, we have also included an MDM bulge component.

In the case of the Milky Way, recent estimates from the RAVE survey yield an escape speed from the solar neighbourhood of $v_{\mathrm{esc}}=544_{-46}^{+64} \mathrm{kms}^{-1}$, and an 
escape to circular speed ratio $v_{\text {esc }} / v_{\text {cir }} \sim 2.5$. If one assumes normal gravity and axially symmetric density structures, the circular speed gives a measure of the amount of matter within the solar circle, while the escape speed gives a measure of the amount of matter outside the solar circle. A measurement of $v_{\text {esc }} / v_{\text {cir }}$ that is significantly larger than $\sqrt{2}$ could then be taken as independent evidence that there is a large proportion of mass outside the solar circle. However, we have shown that our MDM model of the Milky Way yields a similar value $v_{\text {esc }} / v_{\text {cir }} \sim 2.2$, and that given the errors, this particular test cannot be used to establish the presence of a dark halo or to distinguish between the two models at the present time.

This situation is expected to change when the GaiaESO public spectroscopic survey using FLAMES becomes available in 2016 [21]; the survey will yield accurate position and velocity information of a large number of individual stars in the thin and thick discs, the bulge and the halo. Detailed studies of the motions of stars in the solar neighbourhood (as has been done with RAVE) will allow a more precise measurement of the local escape speed. With the new set of data, the study could be extended to establish the radial dependence of the escape velocity, which will provide even stronger constraints on the different dark matter models. We may expect substantial differences under the MDM model in the kinematics of stars in the galactic halo, which may also be measurable in future surveys.

The measurement of escape speeds from discs in external galaxies may also soon become possible. This will require the accumulation of position and velocity data for a large number of individual stars using multiobject optical and infrared spectroscopy with large optical telescopes, along the lines of the recent spectroscopic study of the nearby dwarf irregular galaxy WLM using the VLT and Keck II [26]. Statistical studies of the resulting stellar velocity distributions of a number of such galaxies viewed at different inclinations could then be used to estimate escape speeds and to test the MDM paradigm.

Acknowledgements We wish to thank Ken Freeman for helpful comments and discussion. We also wish to thank the anonymous Referee for his/her constructive suggestions which have greatly improved the manuscript.

\section{References}

1. Begeman, K. G. 1987, PhD thesis, University of Groningen.

2. Begeman K. G., Broeils A. H., Sanders, R. H., Mon. Not.

R. Astron. Soc. 249, 523 (1991)

3. Berezhiani Z., Int. J. Mod. Phys. A, 19, 3775 (2004)
4. Berezhiani Z. et al., J. High Ener. Phys., 7, 83 (2009)

5. Berezhiani Z., Pilo L. \& Rossi N., Eur. Phys. J. C, 70, 305 (2010)

6. Berezhiani Z. \& Nesti F., Eur. Phys. J. C, 72, 1974 (2012)

7. Bienaymé O. et al., Astron. Astrophys, 446, 933, (2006)

8. Blitz L., Fich M., Stark A. A., Astrophys. J. Suppl., 49, 183 (1982)

9. Brownstein J. R. \& Moffat J. W., Astrophys. J. 636, 721 (2006a)

10. Brownstein J. R. \& Moffat J. W., Mon. Not. R. Astron. Soc. 367, 527 (2006b)

11. Burton, W. B. \& Gordon, M. A., Astron. Astrophys 63, 7 (1978)

12. Carignan C., Beaulieu S., Astrophys. J. 347, 760 (1989)

13. Catena R., Ullio P., J. Cosmol. Astropart. Phys. 8, 4 (2010)

14. Ciarcelluti P., Int. J. Mod. Phys. D 19, 2151 (2010)

15. Clemens D. P. (1985), Astrophys. J. 295, 422

16. Flynn C., Fuchs B., Mon. Not. R. Astron. Soc. 270, 471 (1994)

17. Flynn C. et al., Mon. Not. R. Astron. Soc. 372, 1149 (2006)

18. Foot R., Lew H., Volkas R. R., Phys. Lett. B, 272, 67 (1991)

19. Freeman K. C., Astrophys. J. 160, 811 (1970)

20. Gerhard O., Space Sci. Rev. 100, 129 (2002)

21. Gilmore et al., 2012, The Messenger 147.

22. Kobzarev I. Y., Okun L. B., Pomeranchuk I. Y., Sov. J. Nucl. Phys. 3, 837 (1966)

23. Kolb E. W., Seckel D., Turner M. S., Nature 314, 4 (1985)

24. Kuijken K. \& Gilmore G., Mon. Not. R. Astron. Soc. 239, 605 (1989)

25. Lake G., Schommer R. A., van Gorkom J. H., Astron. J. 99, 547 (1990)

26. Leaman, R., Venn, K. A., Brooks, A. M. et al. Ap. J. 750, 33 (2012)

27. Lee T. D., Yang C. N., Phys. Rev. 104, 254 (1956)

28. Milgrom M., Astrophys. J. 270, 365 (1983)

29. McMillan P. J., Mon. Not. R. Astron. Soc. 414, 2446 (2011)

30. Moffat J. W., JCAP, 5, 3 (2005)

31. Navarro J. F., Frenk C. S., White, S. D. M., Mon. Not.

R. Astron. Soc. 275, 720 (1995)

32. Rossi N., Eur. Phys. J. ST 163, 291 (2008)

33. Sanders R. H., McGaugh S. S., ARA\& A, 40, 263 (2002)

34. Smith M. C. et al., Mon. Not. R. Astron. Soc. 379, 755 (2007)

35. Toomre A., Astrophys. J. 138, 385 (1963)

36. Widrow L. M., Pym B., Dubinski J., Astrophys. J. 679, 1239 (2008) 